\title{
Distribution of fermionic and topological observables on the lattice
}

\author{
W. Sakuler, S. Thurner and H. Markum \\ ${ }^{a}$ Institut für Kernphysik, TU Wien, Wiedner Hauptstraße 8-10, A-1040 Vienna, \\ Austria
}

\begin{abstract}
We analyze the topological and fermionic vacuum structure of four-dimensional QCD on the lattice by means of correlators of fermionic observables and topological densities. We show the existence of strong local correlations between the topological charge and monopole density on the one side and the quark condensate, charge and chiral density on the other side. Visualization of individual gauge configurations demonstrates that instantons (antiinstantons) carry positive (negative) chirality, whereas the quark charge density fluctuates in sign within instantons.
\end{abstract}

PACS: 11.15.Ha; 12.38.Gc

Keywords: Lattice gauge theory; topological charge; Abelian monopoles; chiral condensate; quark charge density

\section{Introduction and Theory}

During the last two decades several models have been developed to describe the basic properties of QCD, quark confinement and chiral symmetry breaking. The most popular are the dual superconductor, leading to confinement, and the instanton liquid model, which explains chiral symmetry breaking and solves the $U_{A}(1)$ problem [1]. Both models rely on the existence of topological excitations, monopoles and instantons. Instantons have integer topological charge $Q$ which is associated with the homotopy group $\pi_{3}\left(S U\left(N_{c}\right)\right)=Z$, in four-dimensional Euclidian space. It is related to the zero eigenvalues of the massless Dirac operator $\not D$ via the Atiyah-Singer index theorem [2]:

$$
Q=\operatorname{Index}(\not D)=n_{+}-n_{-},
$$

\footnotetext{
ऋ Supported in part by FWF under Contract No. P11456.
} 
where $n_{+}\left(n_{-}\right)$is the number of zero-modes of positive (negative) chirality. Apart from this famous connection of topology and fermionic degrees of freedom, here we attempt to systematically shed light on the relationship between the sea-quark distribution and topological density. We do this by studying correlators of topological densities with fermionic observables of the form $\bar{\psi} \Gamma \psi$ with $\Gamma=1, \gamma_{4}, \gamma_{5}$. Those quantities are usually referred to as the quark condensate, quark charge density, and the chiral density.

Recently, it was demonstrated that monopole currents which constitute a different topological excitation of compact $\mathrm{SU}(3)$ gauge theory, related to the homotopy group $\pi_{2}\left(S U\left(N_{c}\right) / U(1)^{N_{c}-1}\right)=Z^{N_{c}-1}$, appear preferably in the regions of non-vanishing topological charge density [3,4]. It has been discussed that both instantons and monopoles are related to chiral symmetry breaking [5-7]. In [8] it was shown that zero-modes do not appear in configurations which are not monopole dominated. Here, by interpreting the correlators of monopole densities and fermionic operators, we confirm the local interrelation of monopole and fermionic degrees of freedom.

For the implementation of the topological charge on the lattice there exists no unique discretization. In this work we restrict ourselves to the so-called field theoretic definitions which approximate the topological charge in the continuum,

$$
q(x)=\frac{g^{2}}{32 \pi^{2}} \epsilon^{\mu \nu \rho \sigma} \operatorname{Tr}\left(F_{\mu \nu}(x) F_{\rho \sigma}(x)\right)
$$

in the following ways $[9]$ :

$$
q^{(P, H)}(x)=-\frac{1}{2^{4} 32 \pi^{2}} \sum_{\mu, \ldots= \pm 1}^{ \pm 4} \tilde{\epsilon}_{\mu \nu \rho \sigma} \operatorname{Tr} O_{\mu \nu \rho \sigma}^{(P, H)}
$$

with

$$
O_{\mu \nu \rho \sigma}^{(P)}=U_{\mu \nu}(x) U_{\rho \sigma}(x)
$$

for the plaquette prescription and

$$
\begin{aligned}
O_{\mu \nu \rho \sigma}^{(H)} & =U(x, \mu) U(x+\hat{\mu}, \nu) U(x+\hat{\mu}+\hat{\nu}, \rho) U(x+\hat{\mu}+\hat{\nu}+\hat{\rho}, \sigma) \\
& \times U^{\dagger}(x+\hat{\nu}+\hat{\rho}+\hat{\sigma}, \mu) U^{\dagger}(x+\hat{\rho}+\hat{\sigma}, \nu) U^{\dagger}(x+\hat{\sigma}, \rho) U^{\dagger}(x, \sigma),
\end{aligned}
$$

for the hypercube prescription. We mention here that the topological charges employed are locally gauge invariant, whereas the monopole currents are not. 
The lattice and continuum versions of the theory represent different renormalized quantum field theories, which differ by finite, non-negligible renormalization factors. A simple procedure to get rid of renormalization constants, while preserving physical information contained in lattice configurations, is the cooling method. The cooling procedure systematically reduces quantum fluctuations, and suppresses differences between the different definitions of the topological charge. In our investigation we have employed the so-called "Cabibbo-Marinari method" which consists in a local minimization of the gluonic action in the $\mathrm{SU}(2)$ subgroups of $\mathrm{SU}(3)$ [10].

In order to investigate monopole currents one has to project $S U(N)$ onto its abelian degrees of freedom, such that an abelian $U(1)^{N-1}$ theory remains [11]. This aim can be achieved by various gauge fixing procedures. We employ the so-called maximum abelian gauge which is most favorable for our purposes. For the definition of the monopole currents $m(x, \mu)$ we use the standard method [12]. To extract abelian parallel transporters $u(x, \mu)$ after imposing the maximum abelian gauge one has to perform the decomposition

$$
\tilde{U}(x, \mu)=c(x, \mu) u(x, \mu),
$$

with $(N=3)$

$$
\begin{aligned}
& u(x, \mu)=\operatorname{diag}\left[u_{1}(x, \mu), u_{2}(x, \mu), u_{3}(x, \mu)\right], \\
& u_{i}(x, \mu)=\exp \left[i \arg \tilde{U}_{i i}(x, \mu)-\frac{1}{3} i \phi(x, \mu)\right], \\
& \phi(x, \mu)=\left.\sum_{i} \arg \tilde{U}_{i i}(x, \mu)\right|_{\bmod 2 \pi} \in(-\pi, \pi] .
\end{aligned}
$$

Since the maximum abelian subgroup $U(1)^{N-1}$ is compact, there exist topological excitations. These are color magnetic monopoles which have integer-valued magnetic currents on the links of the dual lattice:

$$
m_{i}(x, \mu)=\frac{1}{2 \pi} \sum_{\square \ni \partial f(x+\hat{\mu}, \mu)} \arg u_{i}(\square),
$$

where $u_{i}(\square)$ denotes a product of abelian links $u_{i}(x, \mu)$ around a plaquette and $f(x+\hat{\mu}, \mu)$ is an elementary cube perpendicular to the $\mu$ direction with origin $x+\hat{\mu}$. The magnetic currents form closed loops on the dual lattice as a consequence of monopole current conservation. From the monopole currents we define the local monopole density as

$$
\rho(x)=\frac{1}{3 \cdot 4} \sum_{\mu, i}\left|m_{i}(x, \mu)\right| .
$$


The local quark condensate $\bar{\psi} \psi(x)$ is a diagonal element of the inverse of the fermionic matrix of the QCD action. Other fermionic operators are obtained by inserting the Euclidian $\gamma_{4}$ and $\gamma_{5}$ matrices. We compute correlation functions between two observables $\mathcal{O}_{1}(x)$ and $\mathcal{O}_{2}(y)$

$$
g(y-x)=\left\langle\mathcal{O}_{1}(x) \mathcal{O}_{2}(y)\right\rangle-\left\langle\mathcal{O}_{1}\right\rangle\left\langle\mathcal{O}_{2}\right\rangle
$$

Since topological objects with opposite sign are equally distributed, we correlate the quark condensate with the square of the topological charge density, and similarly for the other quantities.

\section{Results}

Our simulations were performed for full SU(3) QCD on an $8^{3} \times 4$ lattice with (anti) periodic boundary conditions for the (fermionic) gluonic sector. Applying a standard Metropolis algorithm, we checked that tunneling between sectors of different topological charges occurs at reasonable rates. Dynamical quarks in Kogut-Susskind discretization with $n_{f}=3$ flavors of degenerate mass $m a=0.1$ were taken into account using the pseudofermionic method with 40 updates for the production runs. We performed runs in the confinement phase at inverse gluon coupling $\beta=5.2$, corresponding to a lattice spacing $a \approx 0.2$ $\mathrm{fm}$. This leads to a total extension of the lattice of $1.6 \mathrm{fm}$ and an energy unit of $1 \mathrm{GeV}$. Measurements were taken on 2000 configurations separated by 50 sweeps.

Figure 1 shows results for the correlation functions of Eq. (10) with $\mathcal{O}_{1}$ a local fermionic observable (except in $(\mathrm{d})$ ) and $\mathcal{O}_{2}$ the topological charge density for different numbers of cooling sweeps [13]. The correlations of the two densities are given in lattice units which leads to the small absolute values. The screening masses obtained from fits of the correlators to an exponential are collected in Table 1. The fits were taken over the interval indicated by the lines in Fig. 1. They clearly hint at a linear relation in the logarithmic scale. The statistical errors of the standard deviation in the plots are generally small, leading to relatively small errors in the fit parameters. The extracted quantities have to be interpreted as effective masses and reflect the nonperturbative gluon exchange. Although cooling of quantum fields is necessary to extract topological structure, the exponential slopes of the correlators between the topological charge density and both the local chiral condensate (a) and the absolute value of the quark charge density (b) are nearly cooling-independent. We find that the correlations of the color charge density $\bar{\psi} \psi(x)$ and $\left|\psi^{\dagger} \psi(x)\right|$ with the topological charge density are very similar, both in the slopes and the absolute values. This becomes clear because the quark condensate can be 

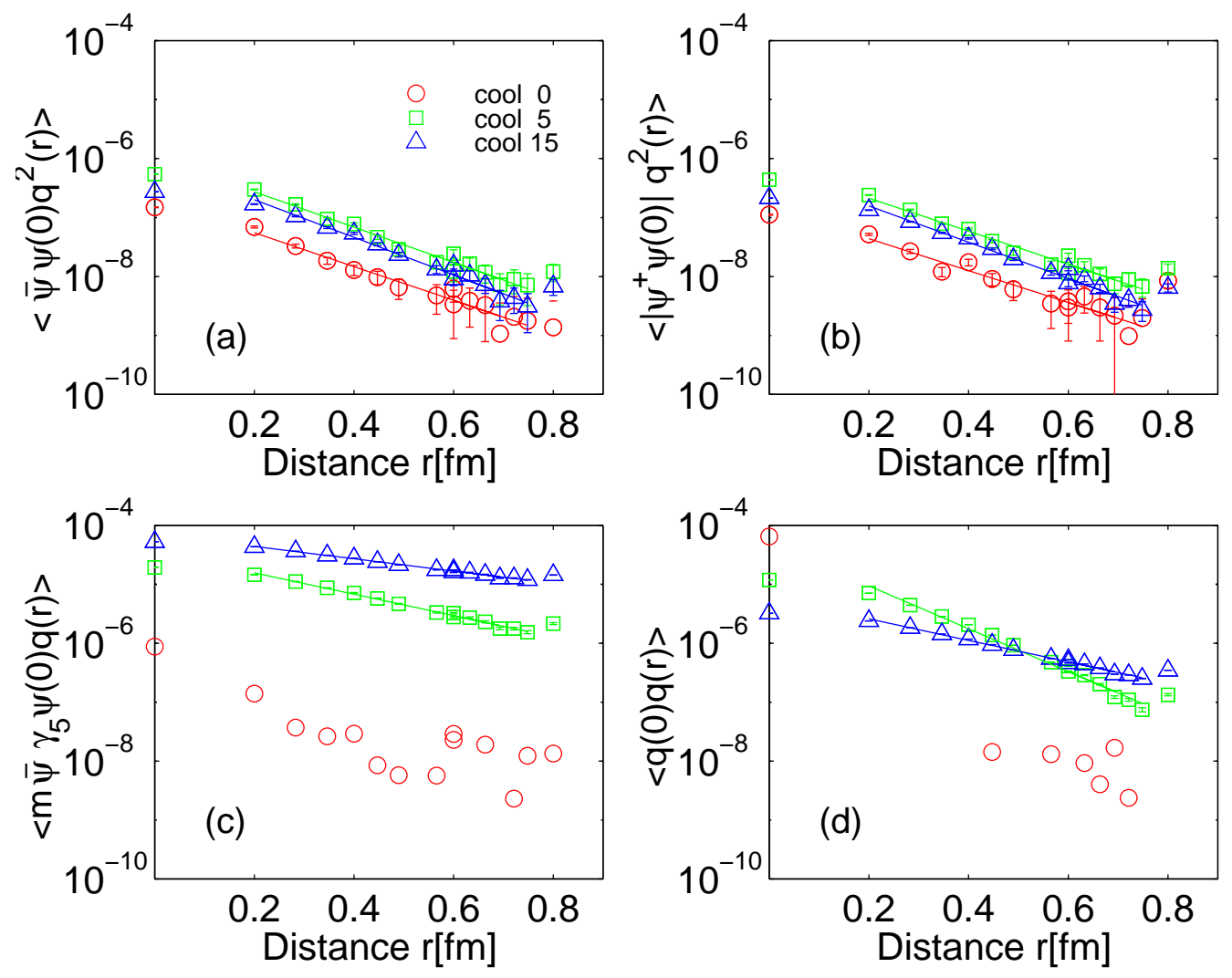

Fig. 1. Correlation functions of the topological charge density with the quark condensate (a), quark charge (b) and chiral density (c), and its autocorrelation (d). For clarity of the plots error bars extending to negative values have been omitted. In the plots (c) and (d) for zero cooling steps some points cannot be resolved or have very large errors.

interpreted as the absolute value of the quark density. However, cooling (or some other kind of smoothing) is inevitable to obtain nontrivial correlations between the chiral density, $\mathcal{O}_{1}=\bar{\psi} \gamma_{5} \psi(x)$, and the topological charge density (c). This can be expected since both quantities are correlated via the anomaly. The topological charge of a gauge field is related to the chiral density of the associated fermion field by

$$
Q=\int q(x) d^{4} x=m \int \bar{\psi} \gamma_{5} \psi(x) d^{4} x .
$$

We have checked that this relation also holds approximately for the corresponding lattice observables on individual configurations. The autocorrelation function of the density of the topological charge $\langle q(0) q(r)>$ (d) should be compared to $<\bar{\psi} \gamma_{5} \psi(0) q(r)>$ (c). If the classical t'Hooft instanton with size $\rho$ is considered, the topological charge density is

$$
q(x) \propto \rho^{4}\left(x^{2}+\rho^{2}\right)^{-4} .
$$


Table 1

\begin{tabular}{lccc}
\hline Correlation & cool 0 & cool 5 & cool 15 \\
\hline $\bar{\psi} \psi(0) q^{2}(r)$ & $1.32(34)$ & $1.38(16)$ & $1.47(16)$ \\
$\left|\psi^{\dagger} \psi(0)\right| q^{2}(r)$ & $1.25(66)$ & $1.29(10)$ & $1.42(09)$ \\
$\bar{\psi} \gamma_{5} \psi(0) q(r)$ & - & $0.84(02)$ & $0.48(01)$ \\
$q(0) q(r)$ & - & $1.67(02)$ & $0.84(01)$ \\
\hline $\bar{\psi} \psi(0) \rho(r)$ & $1.14(10)$ & $1.16(05)$ & $1.27(06)$ \\
$\left|\psi^{\dagger} \psi(0)\right| \rho(r)$ & $1.13(02)$ & $1.08(01)$ & $1.16(01)$ \\
$\left|\bar{\psi} \gamma_{5} \psi(0)\right|^{2} \rho(r)$ & - & $0.98(07)$ & $1.30(10)$ \\
$q^{2}(0) \rho(r)$ & $1.54(47)$ & $1.81(20)$ & $2.41(58)$ \\
\hline
\end{tabular}

Screening masses in $\mathrm{GeV}$ from fits to exponential decays of the various correlators for several cooling steps. The fit ranges are indicated by the lines in the corresponding figures.

On the other hand the corresponding density of fermionic quantities [1]

$$
\bar{\psi} \psi(x) \propto \bar{\psi} \gamma_{5} \psi(x) \propto \rho^{2}\left(x^{2}+\rho^{2}\right)^{-3}
$$

is broader. This behavior is reflected in (c) and (d) where the corresponding correlators are compared. The local relation $q(x)=m \bar{\psi} \gamma_{5} \psi(x)$ does not hold as one might naively expect, the fermionic distributions are of longer range than the instanton profiles. We performed check runs with 400 fermionic updates but did not find significant changes in the correlators.

In Fig. 2 we show the analogous situation to Fig. 1 with $\mathcal{O}_{2}$ being the monopole density. Screening masses from corresponding fits to exponentials are found in Table 1 favoring again an exponential decay of the correlations. As in the case with the topological charge correlators, the monopole correlators are nearly cooling-independent for the local chiral condensate (a) and the modulus of the quark charge density (b). This expresses the strong correlations between the monopole and the topological charge densities themselves [3]. The correlations of the chiral density with the monopole density (c) deserve cooling to resolve a signal. We again compare the topological charge - monopole density correlators (d) to the chiral density results (c). The behavior of their widths is compatible with Eqs. (12) and (13) described above to discuss Fig. 1 (c) and (d).

We now turn to a direct visualization of fermionic densities and topological quantities on individual gauge fields rather than performing gauge averages. We pursue this in the following to get insight into the local interplay of topology with the sea-quark distribution. In a series of papers we found that at clusters of topological charge density, which are identified with instantons, 

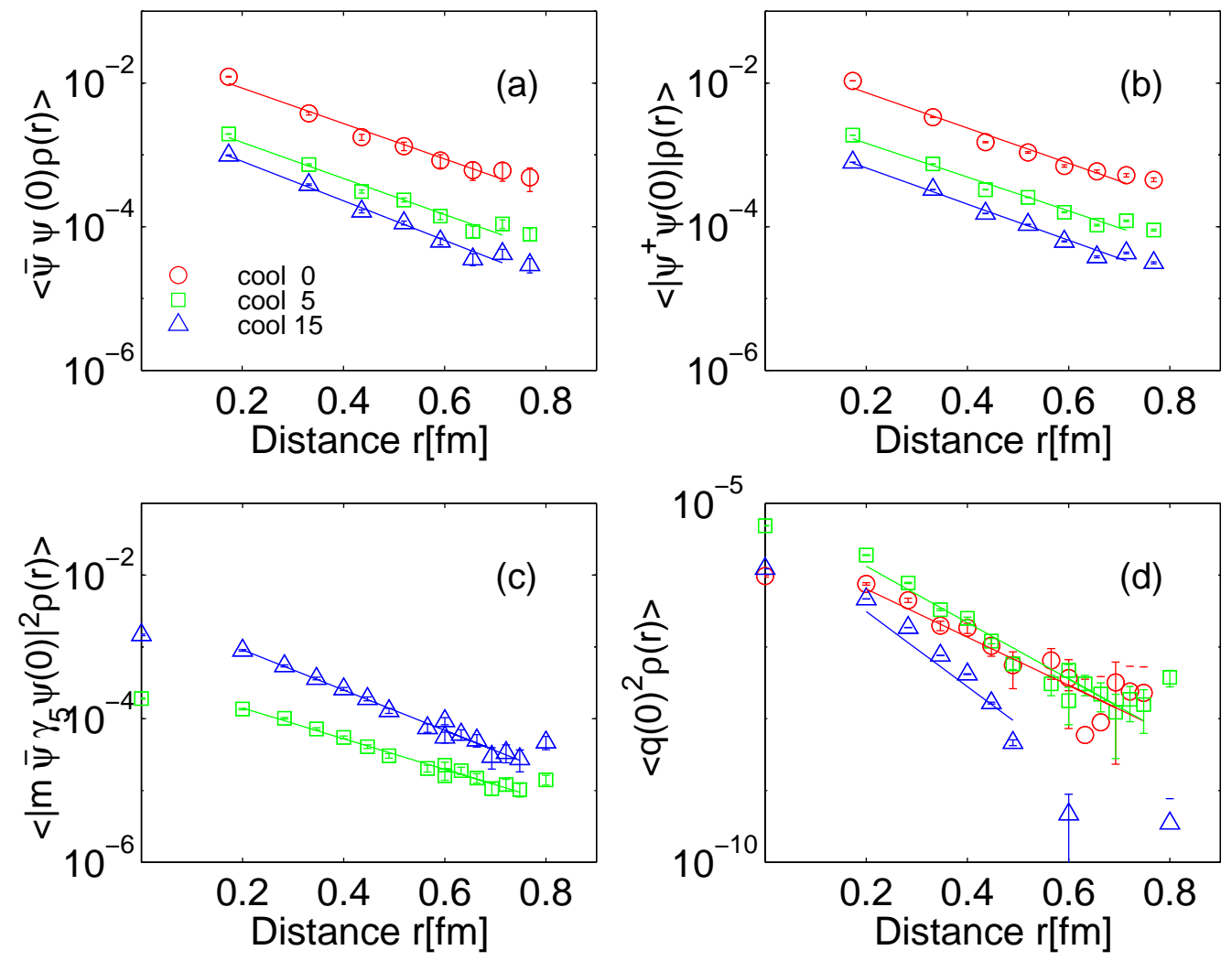

Fig. 2. Correlation functions of the monopole density with the quark condensate (a), quark charge (b) and chiral density (c), and with the topological charge density (d). In the plots (c) and (d) some points cannot be resolved or error bars below zero are not printed.

there are monopole trajectories looping around in almost all cases for $\mathrm{SU}(2)$ and SU(3) gauge theories, with improved action, in the presence of dynamical quarks, even across the deconfinement phase transition [3]. By analyzing dozens of gluon and quark field configurations we obtained the following results. The topological charge is hidden in quantum fluctuations and becomes visible by cooling of the gauge fields. For 0 cooling steps no structure can be seen in $q(x)$, the fermionic observables and the monopole currents, which does not mean the absence of correlations between them. After a few cooling steps clusters of nonzero topological charge density and quark fields are resolved. For more cooling steps both topological charge and quark fields begin to die out and eventually vanish.

In Fig. 3 a typical topologically nontrivial configuration, consisting of an instanton and an antiinstanton, from SU(3) theory with dynamical quarks on the $8^{3} \times 4$ lattice in the confinement phase is shown after 15 cooling steps for fixed time slices. We display the positive/negative topological charge density by white/black dots if the absolute value exceeds certain minimal fluctua- 
(a)

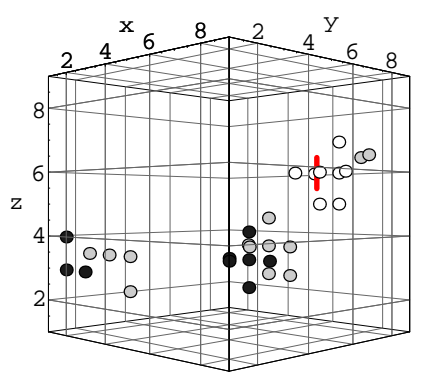

(b)

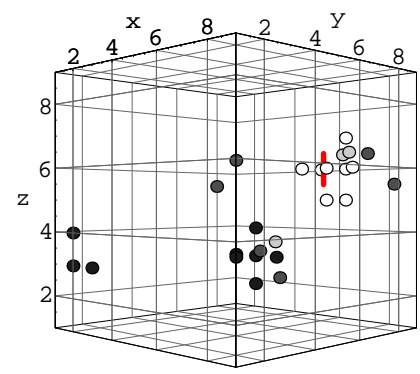

(c)

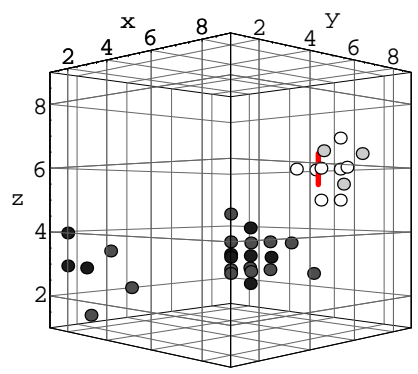

Fig. 3. Aspects of the fermionic field for a fixed time slice in a spatial volume of $(1.6 \mathrm{fm})^{3}$ for a gauge field with an instanton-antiinstanton pair drawn by white and black dots. (a) $\bar{\psi} \psi(x)>0$ (light grey dots) within the instantons. (b) $\psi^{\dagger} \psi(x)$ alternates in sign (light and dark grey dots) in a single instanton. (c) $\bar{\psi} \gamma_{5} \psi(x)$ is positive/negative in instanton/antiinstanton.

tions. Monopole currents are defined in the maximum Abelian projection and only one type is shown by lines. The left 3D plot includes the local chiral condensate $\bar{\psi} \psi(x)$, indicated by light grey dots whenever a certain threshold is exceeded. One clearly sees that both the instanton and antiinstanton are surrounded by a cloud of $\bar{\psi} \psi(x)>0$ [14]. The middle 3D plot exhibits the situation for the quark charge density $\psi^{\dagger} \psi(x)$ indicated by light and dark grey dots depending on the sign of the net color charge excess. One observes that $\psi^{\dagger} \psi(x)$ alternates in sign already in one instanton implying trivial correlations $<\psi^{\dagger} \psi(x) q(y)>=0$ (not shown in Fig. 1). The right 3D plot displays the chiral density $\bar{\psi} \gamma_{5} \psi(x)$ again indicated by light and dark grey dots. One nicely sees that the positive instanton is always surrounded by a lump with $\bar{\psi} \gamma_{5} \psi(x)>0$ and vice versa.

\section{Discussion}

Combining the finding of Fig. 1 and 2 showing that the correlation functions between fermionic and topological quantities are not very sensitive to cooling together with the 3D images in Fig. 3, we conclude that instantons go hand in hand with clusters of $\bar{\psi} \Gamma \psi(x) \neq 0, \Gamma=1, \gamma_{4}, \gamma_{5}$, also in the uncooled QCD vacuum. In summary, our calculations of correlation functions between topological densities and the fermionic observables exhibit exponential behavior. Results for the condensate and the modulus of the quark charge correlators are almost identical, as expected, since the quark condensate reflects the absolute value of the quark charge density. These correlation functions show little 
cooling dependence.

The correlations unambiguously demonstrate that not only the local chiral condensate $\bar{\psi} \psi(x)$ but also the quark charge $\psi^{\dagger} \psi(x)$ and chiral density $\bar{\psi} \gamma_{5} \psi(x)$ take non-vanishing values predominantly in the regions of instantons and monopole loops. Note that for the chiral density this behavior is expected due to the anomaly.

Visualization exhibited that the distribution of sea-quarks is drastically enhanced around centers of nontrivial topology (instantons, monopoles) in Euclidian space-time. Our investigations of full QCD with dynamical quarks rely on lattices of spatial volume of $(1.6 \mathrm{fm})^{3}$ with a relatively large lattice constant of $0.2 \mathrm{fm}$. The correlation functions were also computed in the off-axes directions and show little anisotropy effects. This restauration of rotational invariance is usually taken as a sign of set-in of asymptotic scaling [15]. Nevertheless, it must be emphasized that our results represent the situation on a finite lattice with finite quark mass without the extrapolation to the thermodynamic and chiral limit. However, since all such correlators turned out rather independent of the gauge group and choice of the action etc., we expect that they are generic. We are currently studying fermionic observables along individual monopole trajectories, to further shed light on the puzzle how far monopoles are involved in chiral symmetry breaking.

\section{References}

[1] E.V. Shuryak, Nucl. Phys. B302 (1988) 559; T. Schäfer and E.V. Shuryak, Rev. Mod. Phys. 70 (1998) 323.

[2] M. Atiyah and I. Singer, Ann. Math. 87 (1968) 484.

[3] S. Thurner, H. Markum and W. Sakuler, in Confinement 95, Proceedings of the International Workshop, Osaka, Japan, 1995, edited by H. Toki et al. (World Scientific, 1996) 77; S. Thurner, M. Feurstein, H. Markum and W. Sakuler, Phys. Rev. D54 (1996) 3457; M. Feurstein, H. Markum and S. Thurner, Phys. Lett. B396 (1997) 203.

[4] M.N. Chernodub and F.V. Gubarev, JETP Lett. 62 (1995) 100; R.C. Brower, K.N. Orginos and C.-I Tan, Phys. Rev. D55 (1997) 6313; A. Hart and M. Teper, Phys. Lett. B371 (1996) 261; M. Fukushima, S. Sasaki, H. Suganuma, A. Tanaka, H. Toki and D. Diakonov, Phys. Lett. B399 (1997) 141.

[5] D.I. Dyakonov and V.Yu. Petrov, Phys. Lett. B147 (1984) 351; Sov. Phys. JETP 62 (1985) 204; Nucl. Phys. B272 (1986) 457.

[6] O. Miyamura, Nucl. Phys. B (Proc. Suppl.) 42 (1995) 538. 
[7] S. Thurner, M. Feurstein and H. Markum, Phys. Rev. D56 (1997) 4039; Ph. de Forcrand, M. Garcia Perez, J.E. Hetrick, E. Laermann, J.F. Lagae and I.O. Stamatescu, hep-lat/9810033.

[8] S. Sasaki and O. Miyamura, hep-lat/9810039.

[9] P. Di Vecchia, K. Fabricius, G.C. Rossi and G. Veneziano, Nucl. Phys. B192 (1981) 392; Phys. Lett. B108 (1982) 323; Phys. Lett. B249 (1990) 490.

[10] N. Cabibbo and E. Marinari, Phys. Lett. B119 (1982) 387; M. Teper, Phys. Lett. B162 (1985) 357; B171 (1986) 81; 86; J. Hoek, M. Teper and J. Waterhouse, Phys. Lett. B180 (1986) 112; Nucl. Phys. B288 (1987) 589.

[11] G. 't Hooft, Nucl. Phys. B190 (1981) 455.

[12] A. S. Kronfeld, G. Schierholz and U.-J. Wiese, Nucl. Phys. B293 (1987) 461.

[13] H. Markum, W. Sakuler and S. Thurner, hep-lat/9809155.

[14] S.J. Hands and M. Teper, Nucl. Phys. B347 (1990) 819.

[15] C.B. Lang and C. Rebbi, Phys. Lett. B115 (1982) 137. 